\title{
On subextensive corrections to fluid dynamics from gravity
}

\author{
G.L. Cardoso, ${ }^{a}$ G. Dall'Agata ${ }^{b}$ and V. Grass ${ }^{c, d}$ \\ ${ }^{a}$ CAMGSD, Departamento de Matemática, Instituto Superior Técnico, \\ Av. Rovisco Pais 1, 1049-001 Lisboa, Portugal \\ bDipartimento di Fisica "Galileo Galilei" \& INFN, Sezione di Padova, Università di Padova, \\ Via Marzolo 8, 35131 Padova, Italy \\ ${ }^{c}$ Max-Planck-Institut für Physik, \\ Föhringer Ring 6, 80805 München, Germany \\ ${ }^{d}$ Arnold Sommerfeld Center for Theoretical Physics, \\ Department für Physik, Ludwig-Maximilians-Universität München, \\ Theresienstr. 37, 80333 München, Germany \\ E-mail: gcardoso@math.ist.utl.pt, gianguido.dallagata@pd.infn.it, \\ grass@mppmu .mpg . de
}

ABSTRACT: We use the fluid-gravity correspondence to compute subextensive corrections, proportional to the shear tensor, to the energy-momentum tensor of fluids on three-spheres. The dual configurations we consider are charged black hole solutions of $N=2$ gauged supergravity theories in five dimensions.

KEYWords: Gauge-gravity correspondence, Black Holes in String Theory, AdS-CFT Correspondence 


\section{Contents}

1 Introduction 1

2 Electrically charged static black hole solutions 3

3 Deformed black hole solutions $\quad 6$

3.1 Deformed Schwarzschild black hole solution 8

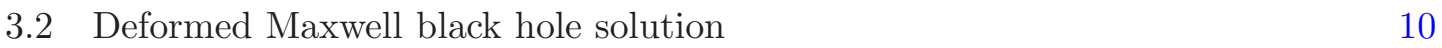

3.3 Deformed black hole solutions supported by scalar fields 13

$\begin{array}{lll}\text { 3.3.1 Two equal charges } & 13\end{array}$

$\begin{array}{lll}3.3 .2 & \text { One charge } & 15\end{array}$

4 Conclusions $\quad 16$

$\begin{array}{ll}\text { A Very special geometry conventions } & 18\end{array}$

B Rotating Maxwell black hole in Eddington-Finkelstein type coordinates 19

C A two-charge rotating STU black hole in Eddington-Finkelstein type co$\begin{array}{lr}\text { ordinates } & \mathbf{2 0}\end{array}$

D Three-charge rotating STU black hole with equal rotation parameters in Eddington-Finkelstein coordinates

E Boundary energy-momentum tensor for the STU black hole solution (3.34)

\section{Introduction}

The conformal fluid-gravity correspondence relates the hydrodynamic regime of strongly coupled four-dimensional conformal field theories to regular black brane solutions in asymptotically $A d S_{5}$ backgrounds [1,2]. The black brane solutions are constructed order by order in a gradient expansion in the bulk, and this gradient expansion is mapped to the hydrodynamic gradient expansion of the fluid's energy-momentum tensor $T_{\mu \nu}$ in the dual boundary theory [3]. The coefficients in the gradient expansion of $T_{\mu \nu}$ are the hydrodynamic transport coefficients that characterize the hydrodynamic properties of the fluid, and these are holographically determined in terms of the black brane solutions.

The gradient expansion of the fluid's energy-momentum tensor contains a term proportional to the shear tensor $\sigma_{\mu \nu}$, with a coefficient $\eta$ that has been computed for various 
conformal fluids dual to black branes, starting with [1]. For these fluids $\eta$ can be expressed in terms of the energy density $\rho$, the pressure $p$ and the diffusion coefficient $D$ as [2]

$$
\eta=(\rho+p) D
$$

For uncharged conformal fluids, $D$ is expressed in terms of the entropy density $s$ of the black brane as $D=\pi^{1 / 3} /(16 s)^{1 / 3}$ (in units where $L=16 \pi G_{5}=1$, where $L$ denotes the curvature radius of $A d S_{5}$ ) [2]. It follows from (1.1) that

$$
\frac{\eta}{s}=\frac{\pi^{1 / 3}}{4^{2 / 3}} \frac{\rho+p}{s^{4 / 3}}
$$

which equals $\eta / s=1 /(4 \pi)$, since for a conformal fluid $\rho=3 p=3 s^{4 / 3} /(4 \pi)^{4 / 3}$. This behavior of $\eta / s$ is also observed for charged fluids, so that for conformal (charged) fluids dual to (charged) black branes, the ratio $\eta / s$ seems to take the universal value $1 /(4 \pi)$ at strong 't Hooft coupling and in the large $N$ limit [1-14].

In this note we will focus on charged black holes in asymptotically $A d S_{5}$ backgrounds, rather than charged black branes. The black hole solutions we consider capture the hydrodynamic expansion of the dual conformal fluid on a three-sphere [15-18]. In contrast to fluids in flat space, the energy of a fluid on a three-sphere is not anylonger a purely extensive quantity $[19,20]$. It contains, in particular, a subextensive part $E_{c}$ which is defined as the violation of the thermodynamic Euler relation [19]. One may ask whether this non-extensivity will result in a correction of the coefficient $\eta$ of the shear tensor and hence in a deviation from the value $\eta / s=1 /(4 \pi)$ for these fluids (at strong 't Hooft coupling and in the large $N$ limit). Consider, for instance, the conformal fluid dual to a Schwarzschild black hole. Its total energy $E$ equals $E=E_{e}+\frac{1}{2} E_{c}$ and the associated density is $\rho=\rho_{e}+\frac{1}{2} \rho_{c}$. Here $E_{e}$ denotes the extensive part of the energy, and its energy density is $\rho_{e}=3 s^{4 / 3} /(4 \pi)^{4 / 3}$. Taking the relation (1.2), which has been derived for flat branes, at face value then suggests that

$$
\frac{\eta}{s}=\frac{1}{4 \pi}\left(1+\frac{\rho_{c}}{2 \rho_{e}}\right)
$$

In this note we will show that (1.3) indeed holds for the conformal fluid dual to a Schwarzschild black hole. For a discussion of a similar effect for fluids on hyperbolic spaces see [21]. In the charged case, the black holes that we consider arise in the so-called STU-model of $N=2$ gauged supergravity in five dimensions. We use the formalism developed in $[3,11,12,15,22,23]$ to construct these electrically charged deformed black hole solutions. We again observe a deviation from the value $\eta / s=1 /(4 \pi)$ in all these cases.

This deviation can be understood as follows. The relation (1.2) was established at first order in the derivative expansion. At this order, $\eta$ has the hydrodynamical interpretation of shear viscosity, since it denotes the coefficient of the shear tensor $\sigma_{\mu \nu}$ in the gradient expansion of $T_{\mu \nu}$. At higher order, however, the fluid's energy-momentum tensor may contain additional higher-derivative terms that are also proportional to the shear tensor. For instance, at cubic order in derivatives, there may be an additional term of the form 
$R \sigma_{\mu \nu}$, where $R$ denotes the curvature scalar of the three-sphere on which the dual fluid lives. Then, combining all the terms proportional to the shear tensor in $T_{\mu \nu}$, yields a shear term with an effective coefficient $\eta$ that will exhibit a departure from the first-order value $s /(4 \pi)$. Whether or not this effective coefficient $\eta$ continues to satisfy relation $(1.2)$ is, a priori, not known.

In [15] it was shown that large rotating black holes in global $A d S_{D}$ spaces are dual to stationary solutions of the relativistic Navier-Stokes equations on $S^{D-2}$. The dual description in terms of fluid dynamics applies when various length scales, namely the one associated with the curvature of the manifold on which the fluid propagates and those describing the variation of the thermodynamic variables, are large compared to the equilibration length scale of the fluid. As shown in [15], this requires taking the horizon radius $R_{H}$ of the dual black hole to be large compared to the AdS radius $R_{\text {AdS }}$. These black holes are non-supersymmetric and are referred to as large black holes. Then, expanding the black hole formulae in a power series in $R_{\text {AdS }} / R_{H}$ results in subleading corrections that show up as corrections in the energy-momentum tensor of the dual fluid. The same continues to hold when considering non-stationary black holes. For large black holes, the subleading corrections in $R_{\mathrm{AdS}} / R_{H}$ in the black hole formulae will contribute to the gradient expansion of the fluid's energy-momentum tensor. An example thereof is the term proportional to $\rho_{c} / \rho_{e}$ in (1.3). For large black holes it constitutes a small correction to the ratio $\eta / s$.

This note is organized as follows. In section 2 we review electrically charged static black hole solutions with spherical horizons of certain five-dimensional $N=2$ gauged supergravity theories. In section 3 we deform these solutions by a slowly varying velocity field and we explain our procedure for determining corrections to $\eta / s$ induced by the curvature $k$ of the fluid's three-sphere. Then we turn to black holes (with up to three equal charges) in the context of the STU-model, and we compute the first correction in $k$ to $\eta / s=1 /(4 \pi)$. Section 4 contains our conclusions. Appendix A summarizes our very special geometry conventions. For the sake of comparison with the deformed solutions in section 3, we summarize various known rotating solutions of the STU-model in appendix B), (C and D. And finally, appendix E summarizes the calculation of the boundary energymomemtum tensor for one of the black hole solutions of the STU-model.

\section{Electrically charged static black hole solutions}

We begin by reviewing the electrically charged static black hole solutions constructed in [24]. These will subsequently be deformed by a non-trivial velocity field. The static solutions of [24] are solutions of five-dimensional $N=2$ gauged supergravity theories obtained by gauging the $\mathrm{U}(1)$ subgroup of the $\mathrm{SU}(2)$-automorphism group of the $N=2$ supersymmetry algebra [25]. The gauging is with respect to a linear combination proportional to $h_{A} A_{M}^{A}$ of $\mathrm{U}(1)$ gauge fields (with constant $h_{A}$ ), and the coupling constant $\mathfrak{g}$ is identified with the inverse of the curvature radius of $A d S_{5}$, i.e. $\mathfrak{g}=L^{-1}$. The relevant part 
of the action reads [25]

$$
\begin{aligned}
16 \pi G_{5} S= & \int d^{5} x \sqrt{-G}\left(R-\mathcal{G}_{i j} \partial_{M} \varphi^{i} \partial^{M} \varphi^{j}-\frac{1}{2} \mathcal{G}_{A B} F_{M N}^{A} F^{B M N}-V_{\mathrm{pot}}\right) \\
& +\frac{\kappa}{\sqrt{3}} \int C_{A B C} F^{A} \wedge F^{B} \wedge A^{C},
\end{aligned}
$$

where $\kappa=-1 /(2 \sqrt{3})$. We denote the five-dimensional spacetime metric by $G_{M N}$. We refer to appendix A for a definition of the various quantities appearing in (2.1).

The static charged black hole solutions we consider are black holes with a spherical horizon. Their line element reads [24]

$$
d s^{2}=G_{M N} d x^{M} d x^{N}=-\mathrm{e}^{-4 \mathrm{U}(r)} p(r) d t^{2}+\mathrm{e}^{2 \mathrm{U}(r)} p^{-1}(r) d r^{2}+\mathrm{e}^{2 \mathrm{U}(r)} r^{2} d \Omega_{3}^{2},
$$

where

$$
p(r)=k-\frac{\mu}{r^{2}}+\frac{\mathrm{e}^{6 U} r^{2}}{L^{2}}, \quad k>0 .
$$

The line element of the three-sphere can be written as

$$
d \Omega_{3}^{2}=g_{i j} d x^{i} d x^{j}=k^{-1}\left(d \theta^{2}+\sin ^{2} \theta d \phi^{2}+\cos ^{2} \theta d \psi^{2}\right),
$$

with $0 \leq \theta \leq \pi / 2,0 \leq \phi<2 \pi, 0 \leq \psi<2 \pi$. The curvature tensor of the three-sphere is $R_{i j}=2 k g_{i j}$, and the associated curvature scalar is $R=6 k$.

These black hole solutions are supported by scalar fields $X^{A}(r)$. They satisfy the relation

$$
X_{A}=\frac{1}{3} \mathrm{e}^{-2 U} H_{A},
$$

where the $H_{A}$ denote harmonic functions given by $H_{A}=h_{A}+q_{A} / r^{2}$. The parameters $q_{A}$ are related to the electric charges and to the mass of the black hole solutions, as we will discuss below. The metric factor $\mathrm{e}^{2 U}$ is given by

$$
\mathrm{e}^{2 U}=\frac{1}{3} H_{A} X^{A}
$$

and its radial derivative $U^{\prime}=d U / d r$ is related to the superpotential $W=h_{A} X^{A}$ by [26],

$$
\mathrm{e}^{2 \mathrm{U}(r)}\left(1+r U^{\prime}\right)=\frac{W}{3} .
$$

We take $h_{A}$ and $q_{A}$ to be positive to ensure that $H_{A}>0$. We also take $X^{A}>0$ so that $\mathrm{e}^{2 U}>0$. We impose the normalization $\mathrm{e}^{2 U}=1$ at $r=\infty$. The asymptotic value of $X_{A}$ is then $\frac{1}{3} h_{A}$. Denoting the asymptotic value of the $X^{A}$ by $h^{A}$, we have $\frac{1}{3} h^{A} h_{A}=1$ in view of real special geometry (see (A.1)). Using $h^{A}$, we introduce the 'dual' superpotential $\tilde{W}$ as

$$
\tilde{W}=h^{A} X_{A},
$$

for later convenience [26]. It asymptotes to $\tilde{W}=1$, while the superpotential $W$ asymptotes to the value $W=3$. 
The mass $M$ of the black hole and its physical electric charges $Q_{A}$ are determined in terms of the parameters $\mu$ and $q_{A}$ as follows [26],

$$
\begin{aligned}
w_{5} M & =\mu+\frac{2}{3} k h^{A} q_{A}, \\
Q_{A} G^{A B} Q_{B} & =k q_{A} G^{A B} q_{B}+\mu q_{A} G^{A B} h_{B},
\end{aligned}
$$

where $w_{5}=16 \pi G_{5} /\left(3 \operatorname{vol}\left(S^{3}\right)\right)$, with $\operatorname{vol}\left(S^{3}\right)=\int d \Omega_{3}$.

Inspection of the line element (2.2) shows that the radius of the three-sphere is $\mathrm{e}^{U} r$ in units of $1 / \sqrt{k}$. It is thus convenient to introduce a new radial coordinate $a=\mathrm{e}^{U} r$. We also introduce the function

$$
f=\mathrm{e}^{-4 U} \frac{p}{a^{2}}=\frac{1}{L^{2}}+\mathrm{e}^{-4 U} \frac{k}{a^{2}}-\mathrm{e}^{-2 U} \frac{\mu}{a^{4}} .
$$

Then, using (2.7), the line element takes the form

$$
d s^{2}=-a^{2} f(a) d t^{2}+9\left(a^{2} f(a) W^{2}(a)\right)^{-1} d a^{2}+a^{2} d \Omega_{3}^{2} .
$$

Next, we introduce Eddington-Finkelstein type coordinates by

$$
v=t+g(a), \quad \frac{d g}{d a}=\frac{3}{W(a) a^{2} f(a)},
$$

so that the line element (2.11) becomes

$$
d s^{2}=-a^{2} f(a) d v^{2}+\frac{6}{W(a)} d v d a+a^{2} d \Omega_{3}^{2} .
$$

Following $[3,15]$, we define boundary coordinates $x^{\mu}=(v, \theta, \phi, \psi)$ and we introduce the associated four-dimensional metric $g_{\mu \nu}=\left(g_{v v}, g_{i j}\right)=\left(-1, g_{i j}\right)$, which will be kept fixed throughout. Then, the static black hole metric (2.13) can be written as

$$
d s^{2}=-a^{2} f(a) u_{\mu} u_{\nu} d x^{\mu} d x^{\nu}-\frac{6}{W(a)} u_{\mu} d x^{\mu} d a+a^{2} P_{\mu \nu} d x^{\mu} d x^{\nu},
$$

where here $u_{\mu}$ denotes the four-vector $u_{\mu}=(-1,0,0,0)$ and where

$$
P_{\mu \nu}=g_{\mu \nu}+u_{\mu} u_{\nu}
$$

The four-vector $u_{\mu}$ denotes the velocity vector of the dual fluid. Indices of boundary tensor quantities will be lowered or raised using the boundary metric $g_{\mu \nu}$ and its inverse $g^{\mu \nu}$, such as, for instance, $u^{\mu}=g^{\mu \nu} u_{\nu}$.

In the following, we set $L=1$ for convenience. Following [22, 23], we introduce the Schouten tensor $S_{\mu \nu}=\frac{1}{2}\left(R_{\mu \nu}-\frac{1}{6} R g_{\mu \nu}\right)$. Here $R_{\mu \nu}$ and $R$ are the four-dimensional Ricci tensor and Ricci scalar computed from the metric $g_{\mu \nu}$. Then, the line element (2.14) can also be expressed as

$$
d s^{2}=-\frac{6}{W(a)} u_{\mu} d x^{\mu} d a+\left[a^{2} g_{\mu \nu}+\mathrm{e}^{-4 U} u_{(\mu} S_{\nu) \lambda} u^{\lambda}+\mathrm{e}^{-2 U} \frac{\mu}{a^{2}} u_{\mu} u_{\nu}\right] d x^{\mu} d x^{\nu}
$$


where $a_{(\mu} b_{\nu)}=a_{\mu} b_{\nu}+a_{\nu} b_{\mu}$. Observe that (2.16) is invariant under the global rescaling $[22,23]$

$$
a \rightarrow \mathrm{e}^{-\chi} a \quad, \quad g_{\mu \nu} \rightarrow \mathrm{e}^{2 \chi} g_{\mu \nu} \quad, \quad u_{\mu} \rightarrow \mathrm{e}^{\chi} u_{\mu} \quad, \quad \mathrm{e}^{U} \rightarrow \mathrm{e}^{U} \quad, \quad \mu \rightarrow \mathrm{e}^{-4 \chi} \mu,
$$

which also implies the rescaling

$$
W \rightarrow W, \quad q_{A} \rightarrow \mathrm{e}^{-2 \chi} q_{A}, \quad k \rightarrow \mathrm{e}^{-2 \chi} k, \quad w_{5} M \rightarrow \mathrm{e}^{-4 \chi} w_{5} M, \quad Q_{A} \rightarrow \mathrm{e}^{-3 \chi} Q_{A} .
$$

Let us now discuss various black hole solutions in the context of the STU-model [27, 28]. This model has three scalar fields $X^{A}$ that are constrained by $X^{1} X^{2} X^{3}=1$, and it allows for two solutions for which $W$ takes a constant value, namely the uncharged Schwarzschild case and the charged Maxwell black hole. In both cases the scalar fields are constant, i.e. $X^{1}=X^{2}=X^{3}=1$, and $W$ takes the value $W=3$. The Maxwell solution is obtained by setting $H_{1}=H_{2}=H_{3}=H=1+q / r^{2}$, in which case $\mathrm{e}^{2 U}=H$. It follows that $a^{2}=r^{2}+q$ and $\mathrm{e}^{-2 U}=1-q / a^{2}$. Inspection of (2.9) yields the mass $M$ and the physical charge $Q$ as $w_{5} M=\mu+2 k q$ and $Q^{2}=k q^{2}+\mu q$. The Schwarzschild solution is obtained by setting $q=0$. In both cases the 'dual' superpotential reads $\tilde{W}=1$.

The STU-model also allows for black hole solutions which are supported by non-trivial scalar fields. An example with two equal charges is obtained by setting $H_{1}=H_{2}=H=$ $1+q / r^{2}$ and $H_{3}=1$, in which case $X^{1}=X^{2}=H^{-1 / 3}, X^{3}=H^{2 / 3}$ as well as $\mathrm{e}^{3 U}=H$. Now the associated mass $M$ and physical charge $Q_{1}=Q_{2}=Q$ read $w_{5} M=\mu+\frac{4}{3} k q$ and $Q^{2}=k q^{2}+\mu q$. On the solution, $W$ is given by $W=2 H^{-1 / 3}+H^{2 / 3}$. The 'dual' superpotential reads $\tilde{W}=\frac{1}{3}\left(H^{-2 / 3}+2 H^{1 / 3}\right)$.

An example with one non-vanishing charge is obtained by setting $H_{1}=H=1+q / r^{2}$ and $H_{2}=H_{3}=1$, in which case $X^{1}=H^{-2 / 3}, X^{2}=X^{3}=H^{1 / 3}$ as well as $\mathrm{e}^{6 U}=H$. Now the associated mass $M$ and physical charge $Q_{1}=Q$ read $w_{5} M=\mu+\frac{2}{3} k q$ and $Q^{2}=k q^{2}+\mu q$. On the solution, $W$ is given by $W=H^{-2 / 3}+2 H^{1 / 3}$. The 'dual' superpotential reads $\tilde{W}=\frac{1}{3}\left(H^{2 / 3}+2 H^{-1 / 3}\right)$.

\section{Deformed black hole solutions}

In the following, we will deform the static solutions described in the previous section by a slowly varying velocity field $u^{\mu}(x)$ of the form

$$
u^{\mu}=\left(1, \epsilon \beta^{\theta}(x), \epsilon \beta^{\phi}(x), \epsilon \beta^{\psi}(x)\right) .
$$

Here we have multiplied the deformation $\beta$ with a small parameter $\epsilon$. Thus, the deformation $u^{i}$ is taken to be small in amplitude. We will work at first order in $\epsilon$. At this order, $u^{\mu}$ satisfies the normalization condition $u^{\mu} u_{\mu}=-1$.

In addition, and following [3], we introduce a counting parameter $\delta$ by performing the rescaling $x^{\mu} \rightarrow \delta x^{\mu}$, so that an expansion in powers of $\delta$ counts covariant derivatives. For instance, the curvature tensor $R_{i j}$ of the three-sphere (which we will call the background curvature tensor in the following) will then come multiplied by a factor $\delta^{2}$. 
The boundary energy-momentum tensor $T_{\mu \nu}$ of the deformed solutions contains a term proportional to the shear tensor $\sigma_{\mu \nu}$, with a coefficient denoted by $\eta$. We are interested in computing corrections to the ratio $\eta / s$ due to the background curvature scalar $R=6 k$. These corrections, if present, give rise to deviations from the value $4 \pi \eta / s=1$, which we write as $4 \pi \eta / s-1=\sum_{p \geq 1} \alpha_{2 p} \delta^{2 p}$. To compute these corrections, we organize the perturbations of the black hole metric in powers of $\epsilon$ and $\delta$. In this note we will only deal with the first subleading correction $\alpha_{2} \delta^{2}$. It corresponds to a term of the type $k \sigma_{\mu \nu}$, and hence of order $\epsilon \delta^{3}$, in the boundary energy-momentum tensor $T_{\mu \nu}$. Thus, we will only keep terms in the perturbed line element that are at most of order $\epsilon \delta^{3}$.

Let us first consider the Schwarzschild case. The static Schwarzschild line element contains a term proportional to the background curvature scalar $R=6 k$. Thus, it contains a term of order $\epsilon^{0} \delta^{2}$. The deformed Schwarzschild solution, on the other hand, contains terms that are of order $\epsilon$ and higher. Its line element has been worked out in [23, 29] at order $\delta^{2}$, and there are only two perturbations that are also of order $\epsilon$, namely the shear tensor $\sigma_{\mu \nu}$ and the perturbation proportional to $u_{\mu} R_{\nu \lambda} u^{\lambda}$. The latter contains the term $u_{t} R_{i j} u^{j}$, which is of order $\epsilon \delta^{2}$. At order $\delta^{3}$, new perturbations will have to be added to the line element. Out of these, only perturbations that are proportional to the shear tensor $\sigma_{\mu \nu}$ can contribute to $\eta$. At order $\epsilon \delta^{3}$ there is only one such term, namely $R \sigma_{\mu \nu}$, which for constant $R$ can be absorbed into the term proportional to $\sigma_{\mu \nu}$ at order $\delta$. Thus, up to order $\epsilon \delta^{3}$, we may restrict the metric perturbations to those involving $\sigma_{\mu \nu}$ and to one particular perturbation of order $\delta^{2}$ associated with the background curvature, namely $u_{\mu} R_{\nu \lambda} u^{\lambda}$.

Now let us discuss deformed charged black hole solutions. In this case there are new perturbations present at each order in $\delta$. For the case of the electrically charged Maxwell black hole, for instance, they were computed up to order $\delta^{2}$ in [11, 12]. Rather than taking all of these new terms into account, we will follow the same strategy as in the Schwarzschild case. Namely, we start with the deformed solution at order $\delta$ and we add one particular perturbation of order $\delta^{2}$ to its line element, namely the one proportional to $u_{\mu} R_{\nu \lambda} u^{\lambda}$.

Now that we have clarified the ingredients we need, we make a solution ansatz using these and we solve the associated equations of motion up to first order in $\epsilon$. We do not truncate the equations of motion. The solution we thus construct at order $\epsilon$ is an exact solution. It is determined in terms of a specific velocity field that is slowly varying in a certain coordinate range. Computing the associated boundary energy-momentum tensor, we find a correction to $\eta / s$ proportional to the background curvature $k$. The addition of further deformations to the line element will, presumably, result in a modified solution that contributes additional terms to $\eta / s$. These new contributions should, however, be qualitatively different from the one we compute here.

The ratio $\eta / s$ should not receive corrections in $\epsilon$, since that would make it depend on the amplitude $\epsilon$ of the velocity field. Indeed, using the results of [23], we have checked that for the Schwarzschild black hole, the second order metric perturbations that are of order $\epsilon^{2} \delta^{2}$ do not contribute to $\eta$.

The solutions we construct at order $\epsilon$ are based on the specific velocity field

$$
u^{\mu}=\left(1,0, \epsilon \beta^{\phi}(\theta), \epsilon \beta^{\psi}(\theta)\right) \text {. }
$$


This velocity field has the special feature that the Weyl connection $\mathcal{A}_{\mu}=u^{\nu} \nabla_{\nu} u_{\mu}-$ $\frac{1}{3}\left(\nabla_{\nu} u^{\nu}\right) u_{\mu}$ introduced in [22] vanishes at order $\epsilon$ (here the covariant derivative $\nabla_{\mu}$ is computed using the boundary metric $\left.g_{\mu \nu}\right)$. In addition, we demand that the mass and the charges of the black hole solution are kept constant at order $\epsilon \delta^{2}$.

In the following, we will first discuss the case of the deformed Schwarzschild black hole and then turn to deformed charged black holes in the STU-model of $N=2$ gauged supergravity.

\subsection{Deformed Schwarzschild black hole solution}

The construction of a black hole solution dual to a conformal fluid starts from a stationary black hole solution in Eddington-Finkelstein coordinates, which then gets deformed by a slowly varying velocity field [3]. Let us consider the static Schwarzschild solution in Eddington-Finkelstein coordinates which, according to (2.16), is given by

$$
d s^{2}=-2 u_{\mu} d x^{\mu} d a+\left[a^{2} g_{\mu \nu}+u_{(\mu} S_{\nu) \lambda} u^{\lambda}+\frac{\mu}{a^{2}} u_{\mu} u_{\nu}\right] d x^{\mu} d x^{\nu},
$$

where $u_{\mu}=(-1,0,0,0)$. Observe that the term proportional to the Schouten tensor is of order $\epsilon^{0} \delta^{2}$. The associated function $f$ reads $f=1+k / a^{2}-\mu / a^{4}$. The event horizon is at $f\left(a_{h}\right)=0$. It will be useful to introduce rescaled variables $\rho=a / a_{h}$ and $m=\mu / a_{h}^{4}$, in terms of which $f$ is given by

$$
f(\rho)=1+\frac{k}{a_{h}^{2} \rho^{2}}-\frac{m}{\rho^{4}} .
$$

The event horizon is at $\rho=1$ and $m$ satisfies $m=1+k / a_{h}^{2}$.

Now we deform (3.3) by taking the velocity field to be non-trivial. The perturbed line element is then written in terms of Weyl covariant combinations [22, 23]. We work at first order in $\epsilon$, and we take the velocity field to be of the form (3.2), for which the Weyl connection vanishes at first order in $\epsilon$. The vanishing of the latter implies that the Weyl-covariantized Schouten tensor $\mathcal{S}_{\mu \nu}$ coincides with the ordinary Schouten tensor $S_{\mu \nu}$.

In general, when deforming the static black hole solution, not only the velocity field $u^{\mu}$ but also the mass $\mu$ becomes a slowly varying function of $x^{\mu}$ [3]. For the velocity field (3.2), inspection of equation (C.1) in [23] shows that $\mu$ remains constant at order $\epsilon \delta^{2}$ provided that $\mathcal{D}^{\nu} \sigma_{\nu \mu}=0$. Here $\mathcal{D}$ denotes the Weyl covariant derivative introduced in [22], and the shear tensor $\sigma_{\mu \nu}$ is defined below. Using this information, we make an ansatz for the line element that captures effects of order $\epsilon \delta^{2}$, and we take $\mu$ to be constant.

We deform (3.3) by adding a term proportional to the shear tensor $\sigma_{\mu \nu}[3,22,23]$,

$$
\sigma_{\mu \nu}=\frac{1}{2}\left(\mathcal{D}_{\mu} u_{\nu}+\mathcal{D}_{\nu} u_{\mu}\right)=\frac{1}{2}\left(P_{\mu \lambda} \nabla^{\lambda} u_{\nu}+P_{\nu \lambda} \nabla^{\lambda} u_{\mu}\right)-\frac{1}{3} P_{\mu \nu} \nabla_{\lambda} u^{\lambda}
$$

where $P_{\mu \nu}=g_{\mu \nu}+u_{\mu} u_{\nu}$. For the velocity field (3.2) this yields $\sigma_{\mu \nu}=\frac{1}{2}\left(\nabla_{\mu} u_{\nu}+\nabla_{\nu} u_{\mu}\right)$ to first order in $\epsilon$. Thus we make the following ansatz for the perturbed line element at order $\epsilon$,

$$
d s^{2}=-2 u_{\mu} d x^{\mu} d a+\left[a^{2} g_{\mu \nu}+u_{(\mu} S_{\nu) \lambda} u^{\lambda}+\frac{\mu}{a^{2}} u_{\mu} u_{\nu}\right] d x^{\mu} d x^{\nu}+2 \frac{a^{2}}{a_{h}} F(a) \sigma_{\mu \nu} d x^{\mu} d x^{\nu}
$$

Here, $F$ has Weyl weight zero, so that (3.6) is invariant under the rescalings (2.17). Observe that according to the counting described above, $\sigma_{\mu \nu}$ is of order $\epsilon \delta$, while $u_{(\mu} S_{\nu) \lambda} u^{\lambda}$ contains the deformation term $u_{(\mu} R_{\nu) \lambda} u^{\lambda}$ which is of order $\epsilon \delta^{2}$. 
Imposing the condition $\mathcal{D}^{\nu} \sigma_{\nu \mu}=0$ we find the following expression for the velocity field,

$$
\begin{aligned}
& \beta^{\phi}(\theta)=\omega_{1}+c_{1}\left(-\frac{1}{4} \log [\cos \theta]+\frac{1}{4} \log [\sin \theta]+\frac{1}{8 \cos ^{2} \theta}\right), \\
& \beta^{\psi}(\theta)=\omega_{2}+c_{2}\left(-\frac{1}{4} \log [\cos \theta]+\frac{1}{4} \log [\sin \theta]-\frac{1}{8 \sin ^{2} \theta}\right),
\end{aligned}
$$

with constants $\omega_{1}, \omega_{2}, c_{1}, c_{2}$. Observe that in obtaining (3.7) we have not resorted to any approximation, i.e. at order $\epsilon(3.7)$ solves $\mathcal{D}^{\nu} \sigma_{\nu \mu}=0$ exactly. The small amplitude approximation, however, breaks down at $\theta=0, \pi / 2$, where the norm of the velocity field diverges. Therefore, we have to restrict the range of $\theta$ to be consistent with the small amplitude expansion. This may be achieved by restricting $\theta$ to be in the range $\lambda<\theta<\pi / 2-\lambda$ with $\epsilon<<\lambda^{2}$.

In case that both the $c_{i}(i=1,2)$ vanish, (3.6) describes an uncharged stationary black hole solution (at order $\epsilon$ ) with $\sigma_{\mu \nu}=0$. In the following, we will be interested in non-stationary solutions, and hence we take at least one of the $c_{i}$ to be non-vanishing. Using (3.7), and inserting the ansatz (3.6) into the Einstein equations of motion, we find that they are satisfied to first order in $\epsilon$ provided that $F$ satisfies the differential equation

$$
\frac{d}{d \rho}\left(\rho^{5} f(\rho) \frac{d}{d \rho} F(\rho)\right)=-\left(3 \rho^{2}+\frac{k}{a_{h}^{2}}\right) .
$$

When solving the Einstein equations, we do not resort to any truncation. Thus, (3.7) and (3.8) yield an exact solution to the Einstein equations at first order in $\epsilon$.

Integrating (3.8) once gives

$$
\rho^{5} f(\rho) \frac{d}{d \rho} F=-\left(\rho^{3}+\frac{k}{a_{h}^{2}} \rho-\zeta\right),
$$

where the integration constant $\zeta$ is set to the value $\zeta=1+k / a_{h}^{2}$ so as to account for the vanishing of $f(\rho)$ at the horizon $\rho=1$. Note that (3.9) can be written as

$$
\frac{d}{d \rho} F=-\frac{\left(\rho^{2}+\rho+\zeta\right)}{\rho(\rho+1)\left(\rho^{2}+\zeta\right)} .
$$

Integrating (3.10) once results in

$$
F(\rho)=\int_{\rho}^{\infty} d u \frac{\left(u^{2}+u+\zeta\right)}{u(u+1)\left(u^{2}+\zeta\right)}
$$

which is well-behaved as long as $\rho>0$. In the limit of large $\rho$ this yields

$$
\frac{F(\rho)}{a_{h}}=\frac{1}{a}-\frac{\eta}{4 a^{4}}
$$

where $\eta=\zeta a_{h}^{3}=a_{h}^{3}+k a_{h}$.

Next we consider the fluid on a three-sphere dual to (3.6). Its energy-momentum tensor $T_{\mu \nu}$ can be computed using standard techniques [30-32], see appendix E. We obtain

$$
16 \pi G_{5}\left\langle T_{\mu \nu}\right\rangle=\frac{1}{4}\left(R_{\alpha \beta} R_{\mu}^{\alpha}{ }_{\nu}{ }_{\nu}-\frac{R^{2}}{12} g_{\mu \nu}\right)+\mu\left(g_{\mu \nu}+4 u_{\mu} u_{\nu}\right)-2 \eta \sigma_{\mu \nu} .
$$


The terms in the first line of this expression denote the contribution to the energymomentum tensor of global $A d S_{5}$ [30,33], while the terms proportional to $\mu$ denote the perfect fluid contribution ( $\mu$ is related to the pressure $p=M /\left(3 \operatorname{vol}\left(S^{3}\right)\right)$ by $\mu=16 \pi G_{5} p$ ). The last term is proportional to the shear tensor. In units where $L=16 \pi G_{5}=1$ the entropy density $s$ of the fluid on a unit three-sphere is $s=\mathcal{S} / \operatorname{vol}\left(S^{3}\right)=4 \pi a_{h}^{3}$, so that the ratio $\eta / s$ reads

$$
\frac{\eta}{s}=\frac{1}{4 \pi}\left(1+\frac{k}{a_{h}^{2}}\right)
$$

\subsection{Deformed Maxwell black hole solution}

Next, let us consider the Maxwell black hole in the context of the STU-model. To this end, we set $X^{1}=X^{2}=X^{3}=1$ as well as $A^{1}=A^{2}=A^{3}=2 A / \sqrt{3}$. Then, from (2.16), we obtain the following line element for the static Maxwell black hole,

$$
d s^{2}=-2 u_{\mu} d x^{\mu} d a+\left[a^{2} g_{\mu \nu}+u_{(\mu} S_{\nu) \lambda} u^{\lambda}+\left(\frac{w_{5} M}{a^{2}}-\frac{Q^{2}}{a^{4}}\right) u_{\mu} u_{\nu}\right] d x^{\mu} d x^{\nu} .
$$

The Maxwell gauge potential reads

$$
A_{\mu}=-\frac{\sqrt{3}}{2} \frac{Q}{a^{2}} u_{\mu} \quad, \quad A_{a}=0,
$$

where $u_{\mu}=(-1,0,0,0)$. The function $f$ in $(2.10)$ reads $f(a)=1+k / a^{2}-w_{5} M / a^{4}+Q^{2} / a^{6}$. The location $a_{h}$ of the outer event horizon is given by the largest positive root of $f(a)$. In terms of the rescaled variables $\rho=a / a_{h}, m=w_{5} M / a_{h}^{4}$ and $\mathcal{Q}=Q / a_{h}^{3}$, the function $f$ is given by

$$
f(\rho)=1+\frac{k}{a_{h}^{2} \rho^{2}}-\frac{m}{\rho^{4}}+\frac{\mathcal{Q}^{2}}{\rho^{6}} .
$$

The outer event horizon is at $\rho=1$ and $m$ satisfies $m=1+k / a_{h}^{2}+\mathcal{Q}^{2}$.

Now we deform the static Maxwell solution by taking the velocity field to be of the form (3.2) with $\beta^{\phi}$ and $\beta^{\psi}$ given by (3.7). We work at first order in $\epsilon$, as before. The results of $[11,12]$ show that at order $\epsilon \delta^{2}$, the electric charge $Q$ can be kept constant when $M$ is constant. In the following, we take both $M$ and $Q$ to be constant.

We construct a solution to the combined Einstein-Maxwell equations of motion as follows. We take the gauge potential to be of the form (3.16) with the velocity field given by (3.7). Inserting this ansatz into the equations of motion, we find that we can solve the combined system exactly at first order in $\epsilon$ with the following line element,

$$
\begin{aligned}
d s^{2}= & -2 u_{\mu} d x^{\mu} d a+\left[a^{2} g_{\mu \nu}+u_{(\mu} S_{\nu) \lambda} u^{\lambda}+\left(\frac{w_{5} M}{a^{2}}-\frac{Q^{2}}{a^{4}}\right) u_{\mu} u_{\nu}\right] d x^{\mu} d x^{\nu} \\
& +\left[2 \sqrt{3} \kappa \frac{Q}{a^{2}} u_{(\mu} l_{\nu)}+2 \frac{a^{2}}{a_{h}} F(a) \sigma_{\mu \nu}\right] d x^{\mu} d x^{\nu}+4 \sqrt{3} \kappa \frac{Q}{a^{4} f(a)} l_{\mu} d x^{\mu} d a,
\end{aligned}
$$

where we recall that $u_{(\mu} l_{\nu)}=u_{\mu} l_{\nu}+u_{\nu} l_{\mu}$, and where $[11,12,33]$

$$
l_{\mu}=\frac{1}{2} \epsilon_{\mu \nu \lambda \sigma} u^{\nu} \mathcal{D}^{\lambda} u^{\sigma}=\frac{1}{2} \epsilon_{\mu \nu \lambda \sigma} u^{\nu} \nabla^{\lambda} u^{\sigma},
$$


with $\epsilon_{\mu \nu \lambda \sigma}=e_{\mu}^{a} e_{\nu}^{b} e_{\lambda}^{c} e_{\sigma}^{d} \epsilon_{a b c d}$. Observe that $l_{\mu}$ and $F(a)$ have Weyl-weight zero, and that the associated terms in (3.18) are of order $\epsilon \delta$, while $u_{(\mu} S_{\nu) \lambda} u^{\lambda}$ contains the deformation term $u_{(\mu} R_{\nu) \lambda} u^{\lambda}$ which is of order $\epsilon \delta^{2}$. The line element (3.18) is invariant under the rescalings (2.17) and (2.18).

The quantity $F$ now satisfies the differential equation

$$
\frac{d}{d \rho}\left(\rho^{5} f(\rho) \frac{d}{d \rho} F(\rho)\right)=-\left(3 \rho^{2}+\frac{k}{a_{h}^{2}}\right)
$$

with $f(\rho)$ given by (3.17). Integrating (3.20) once gives

$$
\rho^{5} f(\rho) \frac{d}{d \rho} F=-\left(\rho^{3}+\frac{k}{a_{h}^{2}} \rho-\zeta\right),
$$

where the integration constant $\zeta$ is set to the value $\zeta=1+k / a_{h}^{2}$ so as to account for the vanishing of $f(\rho)$ at the outer horizon $\rho=1$. Note that (3.21) can be written as

$$
\frac{d}{d \rho} F=-\frac{\rho\left(\rho^{2}+\rho+\zeta\right)}{(\rho+1)\left(\rho^{4}+\zeta \rho^{2}-\mathcal{Q}^{2}\right)} .
$$

Integrating (3.22) once results in

$$
F(\rho)=\int_{\rho}^{\infty} d u \frac{u\left(u^{2}+u+\zeta\right)}{(u+1)\left(u^{4}+\zeta u^{2}-\mathcal{Q}^{2}\right)} .
$$

Here $\rho$ should be taken to be larger than the largest positive root of $u^{4}+\zeta u^{2}-\mathcal{Q}^{2}$ to avoid a singularity in $F(\rho)$. In the limit of large $\rho$ this yields

$$
\frac{F(\rho)}{a_{h}}=\frac{1}{a}-\frac{\eta}{4 a^{4}}
$$

where $\eta=\zeta a_{h}^{3}=a_{h}^{3}+k a_{h}$.

The line element (3.18) is not in the customary gauge $g_{a \mu}=-u_{\mu}$ [23]. It can be brought into this gauge by the following coordinate transformation at order $\epsilon$,

$$
d x^{\mu} \rightarrow d x^{\mu}-h(a) l^{\mu} d a-\left(\int^{a} h(b) d b\right) d l^{\mu}
$$

where $h(a)=2 \sqrt{3} \kappa Q /\left(a^{6} f(a)\right)$. Here the term proportional to $l^{\mu}$ is of order $\epsilon \delta$, while the term proportional to $d l^{\mu}$ is of order $\epsilon \delta^{2}$. The resulting line element is then regular at the outer horizon $f\left(a_{h}\right)=0$ of the undeformed static black hole solution.

In the stationary case, the velocity field has the form (3.7) with $c_{i}=0$. Due to the curvature $k$ of the background, $l^{\mu}$ is non-vanishing but constant and given by $l^{\mu}=$ $\sqrt{k}\left(0,0,-\omega_{2},-\omega_{1}\right)$. Then the second term in (3.25) vanishes, and the line element takes the form

$$
\begin{gathered}
d s^{2}=-2 u_{\mu} d x^{\mu} d a+\left[a^{2} g_{\mu \nu}+u_{(\mu} S_{\nu) \lambda} u^{\lambda}+\left(\frac{w_{5} M}{a^{2}}-\frac{Q^{2}}{a^{4}}\right) u_{\mu} u_{\nu}\right. \\
\left.+2 \sqrt{3} \kappa \frac{Q}{a^{2}} u_{(\mu} l_{\nu)}\right] d x^{\mu} d x^{\nu}
\end{gathered}
$$


in the gauge $g_{a \mu}=-u_{\mu}$. It is straightforward to relate this line element to the usual one [34] written in Boyer-Lindquist type coordinates, to linear order in $\omega_{1}$ and $\omega_{2}$, see appendix B.

Next we compute the associated boundary energy-momentum tensor $T_{\mu \nu}$ of the fluid, see appendix E. We obtain

$$
\begin{aligned}
16 \pi G_{5}\left\langle T_{\mu \nu}\right\rangle= & \frac{1}{4}\left(R_{\alpha \beta} R_{\mu}^{\alpha}{ }^{\beta}{ }_{\nu}-\frac{R^{2}}{12} g_{\mu \nu}\right) \\
& +w_{5} M\left(g_{\mu \nu}+4 u_{\mu} u_{\nu}\right)+8 \sqrt{3} \kappa Q u_{(\mu} l_{\nu)}-2 \eta \sigma_{\mu \nu} .
\end{aligned}
$$

In units where $L=16 \pi G_{5}=1$ (using that the entropy density $s$ of the fluid on a unit three-sphere is $\left.s=\mathcal{S} / \operatorname{vol}\left(S^{3}\right)=4 \pi a_{h}^{3}\right)$, the ratio $\eta / s$ reads

$$
\frac{\eta}{s}=\frac{1}{4 \pi}\left(1+\frac{k}{a_{h}^{2}}\right)
$$

as in the Schwarzschild case. We note that the correction to $\eta / s=1 /(4 \pi)$ is determined by the coefficient of the $u R u$-term in the line element (3.18).

In the stationary case, where $\sigma_{\mu \nu}=0, T_{\mu \nu}$ takes the form given in [35]. It contains additional non-dissipative terms proportional to $l_{\mu}$ associated with the rotation of the fluid in a background of constant curvature $k$.

In $[11,12]$ the authors constructed charged black brane solutions up to order $\delta^{2}$. At order $\delta$, their solution is based on the gauge field

$$
A_{\mu}=-\frac{\sqrt{3} Q}{2 a^{2}}\left(u_{\mu}-2 \sqrt{3} \kappa \frac{Q}{w_{5} M} l_{\mu}\right) \quad, \quad A_{a}=0 .
$$

For the sake of comparison, let us construct a black hole solution based on (3.29) with the velocity field given by (3.7). Inserting this ansatz into the equations of motion, we find that we can solve them exactly at first order in $\epsilon$ with the following line element,

$$
\begin{aligned}
d s^{2}= & -2 u_{\mu} d x^{\mu} d a+\left[a^{2} g_{\mu \nu}+u_{(\mu} S_{\nu) \lambda} u^{\lambda}+\left(\frac{w_{5} M}{a^{2}}-\frac{Q^{2}}{a^{4}}\right) u_{\mu} u_{\nu}\right] d x^{\mu} d x^{\nu} \\
& +\left[-\frac{6 \kappa^{2} Q^{2}}{w_{5} M a^{2}} u_{(\mu} R_{\nu) \lambda} u^{\lambda}+\frac{2 \sqrt{3} \kappa Q^{3}}{w_{5} M a^{4}} u_{(\mu} l_{\nu)}+2 \frac{a^{2}}{a_{h}} F(a) \sigma_{\mu \nu}\right] d x^{\mu} d x^{\nu} \\
& +\left[\frac{4 \sqrt{3} \kappa Q^{3}}{w_{5} M a^{6} f} l_{\mu}-\frac{12 \kappa^{2} Q^{2}}{w_{5} M a^{4} f} R_{\mu \lambda} u^{\lambda}\right] d x^{\mu} d a
\end{aligned}
$$

with $l_{\mu}$ defined as in (3.19). The quantity $F$ satisfies the differential equation (3.20). The line element (3.30) is invariant under the rescalings (2.17) and (2.18). It is again not in the customary gauge $g_{a \mu}=-u_{\mu}$ [23]. It can be brought into this gauge by the coordinate transformation (3.25) at order $\epsilon$. The resulting line element is then regular at the outer horizon $f\left(a_{h}\right)=0$ of the undeformed static black hole solution.

One may ask whether the two line elements (3.18) and (3.30) can be transformed into each other. The associated gauge fields are related by the shift $u^{\mu} \rightarrow u^{\mu}-2 \sqrt{3} \kappa \frac{Q}{w_{5} M} l^{\mu}$. Applying this shift to the line element (3.18) induces terms that are of order $\epsilon \delta^{3}$. The 
resulting line element thus has terms of different order in $\delta$ than the line element (3.30). Matching of these two line elements is thus only expected to occur when the full set of $\epsilon \delta^{3}$-terms is taken into account. However, in the stationary case $\left(c_{i}=0\right)$, the solution (3.18) is mapped into (3.30) at order $\epsilon$ by the shift of $u^{\mu}$ described above, under which $l_{i} \rightarrow l_{i}-\sqrt{3} \kappa \frac{Q}{w_{5} M} R_{i j} u^{j}$. The two line elements are then identical in the gauge $g_{a \mu}=-u_{\mu}$, as expected.

Let us now compare the line element (3.30) with the one obtained in [11, 12]. Since the gauge field (3.29) is at most of order $\epsilon \delta$, the comparison is only meaningful up to this order. Since the terms in (3.30) proportional to $R_{\mu \nu}$ are of order $\epsilon \delta^{2}$ they should be dropped in the comparison. Then, by going into the gauge $g_{a \mu}=-u_{\mu}$ via the coordinate transformation (3.25) (and dropping the term proportional to $d l^{\mu}$ which is also of order $\left.\epsilon \delta^{2}\right)$ we find that the line element (3.30) goes over into the one obtained in [11, 12].

Computing the associated boundary energy-momentum tensor $T_{\mu \nu}$ we obtain

$$
\begin{aligned}
16 \pi G_{5}\left\langle T_{\mu \nu}\right\rangle= & \frac{1}{4}\left(R_{\alpha \beta} R_{\mu}^{\alpha{ }^{\beta}{ }_{\nu}}-\frac{R^{2}}{12} g_{\mu \nu}\right) \\
& +w_{5} M\left(g_{\mu \nu}+4 u_{\mu} u_{\nu}\right)-\frac{24 \kappa^{2} Q^{2}}{w_{5} M} u_{(\mu} R_{\nu) \lambda} u^{\lambda}-2 \eta \sigma_{\mu \nu},
\end{aligned}
$$

with $\eta / s$ given by (3.28). It contains non-dissipative terms proportional to the background curvature tensor $R_{\mu \nu}$. In the stationary case, the boundary energy-momentum tensor (3.27) matches (3.31) under the constant shift $u^{\mu} \rightarrow u^{\mu}-2 \sqrt{3} \kappa \frac{Q}{w_{5} M} l^{\mu}$ discussed above.

\subsection{Deformed black hole solutions supported by scalar fields}

Next, we consider black hole solutions in the STU-model that are supported by non-trivial scalar fields, and that carry either one or two non-vanishing charges. In the two-charge case, we take the charges to be equal, for simplicity. We deform the static solutions in the manner described above. We find that the scalar fields need not be deformed at order $\epsilon$.

\subsubsection{Two equal charges}

We begin by first considering the case of two equal charges. The line element of the static solution is given by (2.14) and the gauge potentials and scalar fields are

$$
\begin{array}{ll}
A_{\mu}^{1}=A_{\mu}^{2}=-\frac{Q}{a^{2}} H^{-\frac{1}{3}} u_{\mu}, & A_{\mu}^{3}=0, \quad A_{a}^{i}=0, \quad i=1,2,3, \\
X^{1}=X^{2}=H^{-\frac{1}{3}}, & X^{3}=H^{\frac{2}{3}}
\end{array}
$$

where $u_{\mu}=(-1,0,0,0)$. We refer to the end of section 2 for a definition of the various quantities involved. The function $f(a)$ appearing in (2.14), when expressed in terms of the rescaled coordinates $\rho=a / a_{h}$, reads

$$
f(\rho)=1+\mathrm{e}^{-4 U} \frac{k}{a_{h}^{2} \rho^{2}}-\mathrm{e}^{-2 U} \frac{m}{\rho^{4}} \quad, \quad m=\frac{\mu}{a_{h}^{4}}=\left(1+\frac{k}{a_{h}^{2}} \mathrm{e}^{-4 \mathrm{U}\left(a_{h}\right)}\right) \mathrm{e}^{2 \mathrm{U}\left(a_{h}\right)} .
$$

The outer horizon is at $\rho=1$. 
We perturb this static solution by again taking the velocity field to have the form (3.2) and (3.7). This results in a modification of the line element, and it also induces a nonvanishing $A^{3}$. We find that at first order in $\epsilon$ (but no approximation otherwise) the combined system of equations of motion is solved by

$$
\begin{aligned}
d s^{2}= & -a^{2} f(a) u_{\mu} u_{\nu} d x^{\mu} d x^{\nu}-\frac{6}{W(a)} u_{\mu} d x^{\mu} d a+a^{2} P_{\mu \nu} d x^{\mu} d x^{\nu} \\
& +\frac{1}{2} H^{-\frac{1}{3}} u_{(\mu} R_{\nu) \lambda} u^{\lambda} d x^{\mu} d x^{\nu}+2 \frac{a^{2}}{a_{h}} F(a) \sigma_{\mu \nu} d x^{\mu} d x^{\nu} \\
A_{\mu}^{1}= & A_{\mu}^{2}=-\frac{Q}{a^{2}} H^{-\frac{1}{3}} u_{\mu}, \quad A_{\mu}^{3}=-\frac{q}{a^{2}} H^{\frac{2}{3}} l_{\mu}, \quad A_{a}^{i}=0, \quad i=1,2,3
\end{aligned}
$$

with the scalar fields given as in (3.32). Here $l_{\mu}$ and the velocity field are again given by (3.19) and (3.7), respectively. The stationary limit of this solution can be easily related to the solution found in [36] written in Boyer-Lindquist type coordinates, to linear order in rotation parameters (see appendix $\mathrm{C}$ ).

The quantity $F$ now satisfies the differential equation

$$
\frac{1}{3} \frac{d}{d \rho}\left(\rho^{5} W(\rho) f(\rho) \frac{d}{d \rho} F(\rho)\right)=-\left(3 \rho^{2}+\frac{k}{a_{h}^{2}} \mathrm{e}^{-U}\left(1-U^{\prime} \rho\right)\right)
$$

where $U^{\prime}=d U / d \rho$, with $\mathrm{e}^{3 U}=H$. We note the appearance of the superpotential $W(a)$ on the left hand side, which was constant $(W(a)=3)$ in both the Schwarzschild and the Maxwell case. The right hand side of (3.35) can be easily integrated by noting that the second term is a total derivative,

$$
\mathrm{e}^{-U}\left(1-U^{\prime} \rho\right) d \rho=d\left(\rho \mathrm{e}^{-U}\right)
$$

Thus, integrating (3.35) once gives

$$
\frac{1}{3} \rho^{5} W(\rho) f(\rho) \frac{d}{d \rho} F(\rho)=-\left(\rho^{3}+\frac{k}{a_{h}^{2}} \mathrm{e}^{-U} \rho-\zeta\right) .
$$

The integration constant $\zeta$ is set to the value $\zeta=1+\left(k \mathrm{e}^{-\mathrm{U}\left(a_{h}\right)}\right) / a_{h}^{2}$ to allow for the vanishing of (3.35) at the outer horizon $\rho=1$, where $f=0$. Then, integrating (3.37) once results in

$$
F(\rho)=\int_{\rho}^{\infty} \frac{d u}{W(u)} \frac{3\left(u^{3}+u(\zeta-1) \mathrm{e}^{\mathrm{U}\left(a_{h}\right)} \mathrm{e}^{-U}-\zeta\right)}{u^{5}+u^{3}(\zeta-1) \mathrm{e}^{\mathrm{U}\left(a_{h}\right)} \mathrm{e}^{-4 U}-u\left(\mathrm{e}^{2 \mathrm{U}\left(a_{h}\right)}+(\zeta-1) \mathrm{e}^{-\mathrm{U}\left(a_{h}\right)}\right) \mathrm{e}^{-2 U}}
$$

For large $\rho$ we have $\mathrm{e}^{3 U}=H \approx 1+q /\left(a_{h}^{2} \rho^{2}\right)$, and hence we obtain

$$
\frac{F(\rho)}{a_{h}}=\frac{1}{a}-\frac{\eta}{4 a^{4}},
$$

where $\eta=\zeta a_{h}^{3}=a_{h}^{3}+k \mathrm{e}^{-\mathrm{U}\left(a_{h}\right)} a_{h}$. 
Computing the associated boundary energy-momentum tensor we obtain (see appendix E)

$$
\begin{aligned}
16 \pi G_{5}\left\langle T_{\mu \nu}\right\rangle= & \frac{1}{4}\left(R_{\alpha \beta} R_{\mu}^{\alpha}{ }^{\beta}{ }_{\nu}-\frac{R^{2}}{12} g_{\mu \nu}\right) \\
& +w_{5} M\left(g_{\mu \nu}+4 u_{\mu} u_{\nu}\right)-\frac{2 q}{3} u_{(\mu} R_{\nu) \lambda} u^{\lambda}-2 \eta \sigma_{\mu \nu} .
\end{aligned}
$$

It contains a non-dissipative term proportional to the background curvature tensor $R_{\mu \nu}$. In units where $L=16 \pi G_{5}=1$, the ratio $\eta / s$ reads

$$
\frac{\eta}{s}=\frac{1}{4 \pi}\left(1+\frac{k \mathrm{e}^{-\mathrm{U}\left(a_{h}\right)}}{a_{h}^{2}}\right) .
$$

We note that the correction to $\eta / s=1 /(4 \pi)$ is determined by the coefficient of the $u R u$ term in the line element (3.34).

\subsubsection{One charge}

Next we consider the case of one non-vanishing charge. Proceeding as before, i.e. taking the velocity field to be given by (3.7), we find that at first order in $\epsilon$ (but no approximation otherwise) the perturbed solution to the combined system of equations of motion is given by

$$
\begin{aligned}
d s^{2}= & -a^{2} f(a) u_{\mu} u_{\nu} d x^{\mu} d x^{\nu}-\frac{6}{W(a)} u_{\mu} d x^{\mu} d a+a^{2} P_{\mu \nu} d x^{\mu} d x^{\nu} \\
& +\frac{1}{2} H^{\frac{1}{3}} u_{(\mu} R_{\nu) \lambda} u^{\lambda} d x^{\mu} d x^{\nu}+2 \frac{a^{2}}{a_{h}} F(a) \sigma_{\mu \nu} d x^{\mu} d x^{\nu} \\
A_{\mu}^{1}= & -\frac{Q}{a^{2}} H^{-\frac{2}{3}} u_{\mu} \quad A_{\mu}^{2}=A_{\mu}^{3}=0 \quad A_{a}^{i}=0 \quad i=1,2,3 \\
X^{1}= & H^{-\frac{2}{3}} \quad X^{2}=X^{3}=H^{\frac{1}{3}}
\end{aligned}
$$

The stationary limit of this solution can be related to the solution found in [37, 38], to linear order in rotation parameters (see appendix D). The quantity $F$ satisfies the differential equation

$$
\frac{1}{3} \frac{d}{d \rho}\left(\rho^{5} W(\rho) f(\rho) \frac{d}{d \rho} F(\rho)\right)=-\left(3 \rho^{2}+\frac{k}{a_{h}^{2}} \mathrm{e}^{2 U}\left(1+2 U^{\prime} \rho\right)\right)
$$

where $U^{\prime}=d U / d \rho$, with $\mathrm{e}^{6 U}=H$. The right hand side of (3.43) can be easily integrated by noting that the second term is a total derivative,

$$
\mathrm{e}^{2 U}\left(1+2 U^{\prime} \rho\right) d \rho=d\left(\rho \mathrm{e}^{2 U}\right)
$$

Integrating (3.43) once gives

$$
\frac{1}{3} \rho^{5} W(\rho) f(\rho) \frac{d}{d \rho} F(\rho)=-\left(\rho^{3}+\frac{k}{a_{h}^{2}} \mathrm{e}^{2 U} \rho-\zeta\right) .
$$


The integration constant $\zeta$ is set to the value $\zeta=1+\left(k \mathrm{e}^{2 \mathrm{U}\left(a_{h}\right)}\right) / a_{h}^{2}$ to allow for the vanishing of (3.43) at the outer horizon $\rho=1$, since $f=0$ there. Then, integrating (3.45) once results in

$$
F(\rho)=\int_{\rho}^{\infty} \frac{d u}{W(u)} \frac{3\left(u^{3}+u(\zeta-1) \mathrm{e}^{-2 \mathrm{U}\left(a_{h}\right)} \mathrm{e}^{2 U}-\zeta\right)}{u^{5}+u^{3}(\zeta-1) \mathrm{e}^{-2 \mathrm{U}\left(a_{h}\right)} \mathrm{e}^{-4 U}-u\left(\mathrm{e}^{2 \mathrm{U}\left(a_{h}\right)}+(\zeta-1) \mathrm{e}^{-4 \mathrm{U}\left(a_{h}\right)}\right) \mathrm{e}^{-2 U}}
$$

For large $\rho$ we have $\mathrm{e}^{6 U}=H \approx 1+q /\left(a_{h}^{2} \rho^{2}\right)$, and hence we obtain

$$
\frac{F(\rho)}{a_{h}}=\frac{1}{a}-\frac{\eta}{4 a^{4}}
$$

where now $\eta=\zeta a_{h}^{3}=a_{h}^{3}+k \mathrm{e}^{2 \mathrm{U}\left(a_{h}\right)} a_{h}$.

Computing the associated boundary energy-momentum tensor yields

$$
\begin{aligned}
16 \pi G_{5}\left\langle T_{\mu \nu}\right\rangle= & \frac{1}{4}\left(R_{\alpha \beta} R_{\mu}^{\alpha}{ }_{\nu}{ }_{\nu}-\frac{R^{2}}{12} g_{\mu \nu}\right) \\
& +w_{5} M\left(g_{\mu \nu}+4 u_{\mu} u_{\nu}\right)+\frac{2 q}{3} u_{(\mu} R_{\nu) \lambda} u^{\lambda}-2 \eta \sigma_{\mu \nu} .
\end{aligned}
$$

In units where $L=16 \pi G_{5}=1$, the ratio $\eta / s$ reads

$$
\frac{\eta}{s}=\frac{1}{4 \pi}\left(1+\frac{k \mathrm{e}^{2 \mathrm{U}\left(a_{h}\right)}}{a_{h}^{2}}\right)
$$

We note that the correction to $\eta / s=1 /(4 \pi)$ is determined by the coefficient of the $u R u$ term in the line element (3.42).

\section{Conclusions}

As mentioned in the introduction, the energy of a perfect fluid on a three-sphere dual to a static black hole is not a purely extensive quantity $[19,20]$. It contains a subextensive piece $E_{c}$ which is defined as the violation of the thermodynamic Euler relation. In the context of $N=2$ gauged supergravity theories, the ratio of $E_{c}$ and the extensive part $E_{e}$ of the energy, when expressed in terms of black hole data, reads (in units where $L=16 \pi G_{5}=1$ ) [26]

$$
\frac{E_{c}}{E_{e}}=6 k \frac{\tilde{W}_{h}}{W_{h}}\left(\frac{4 \pi}{s}\right)^{2 / 3}
$$

where $s=\mathcal{S} / \operatorname{vol}\left(S^{3}\right)$, and where $\tilde{W}_{h}$ and $W_{h}$ denote the superpotentials evaluated at the horizon. The Schwarzschild and the Maxwell black hole both satisfy $W_{h}=3, \tilde{W}_{h}=1$. For these two black holes, the ratio $\eta / s$ in (3.28) can be written as

$$
\frac{\eta}{s}=\frac{1}{4 \pi} \frac{E_{e}+\frac{1}{2} E_{c}}{E_{e}}=\frac{1}{4 \pi}\left(1+3 k \frac{\tilde{W}_{h}}{W_{h}}\left(\frac{4 \pi}{s}\right)^{2 / 3}\right)
$$

and thus it takes the form (1.3). 
The ratio displayed in (4.2) takes a form that is written in manifest $N=2$ language and that could, a priori, be applicable to any black hole in an $N=2$ model. However, inspection of the two-charge result (3.41) and of the one-charge result (3.49) shows that they are not simply captured by (4.2). These two cases involve non-trivial scalar fields, and it is conceivable that additional terms involving these will have to be added to (4.2) in order to obtain an expression that is valid for a general $N=2$ model.

Let us now discuss the diffusion coefficient $D$, defined as in (1.1). Let us first consider the Schwarzschild case, for which (4.2) implies that the ratio $D=\eta /(\rho+p)=3 \eta /(4 \rho)$ equals $D=\pi^{1 / 3} /\left(4^{2 / 3} s^{1 / 3}\right)$, as in the black brane case (1.2). Thus, when viewed as a function of $s, D$ does not change its functional form. On the other hand, if $D$ is viewed as a function of the temperature (the energy), then $D$ will change its functional form due to the subextensive contribution $E_{c} \propto k$ to the total energy, i.e. $D$ will not anylonger be simply given in terms of the inverse of the temperature. Either way, $\eta=D(\rho+p)$ will receive a correction proportional to $E_{c} \propto k$ (see (4.1)).

Next, let us consider the Maxwell case. Viewing $D$ as a function of $s$, we find that $D$ is not anylonger given by $D=\pi^{1 / 3} /\left(4^{2 / 3} s^{1 / 3}\right)$. This can be understood as follows. The total energy of the system is not simply $E_{e}+\frac{1}{2} E_{c}$, but rather $E_{e}+\frac{1}{2} E_{c}+\frac{1}{2} Q_{A} \phi_{h}^{A}$, where $\phi_{h}^{A}$ denote the electrostatic potentials at the horizon [26]. The contribution $Q_{A} \phi_{h}^{A}$ is a subextensive contribution that is distinct from the subleading contribution $E_{c}$. The former is proportional to the square of the charge, while the latter is proportional to $k$. Using (4.2), we find that the diffusion coefficient $D$ is proportional to the ratio $\left(E_{e}+\right.$ $\left.\frac{1}{2} E_{c}\right) /\left(E_{e}+\frac{1}{2} E_{c}+\frac{1}{2} Q_{A} \phi_{h}^{A}\right)$. At first order in $E_{c}$, the correction proportional to $k$ cancels out, while the term proportional to $Q_{A} \phi_{h}^{A}$ changes the functional dependence of $D$ on $s$, an effect already observed in [7] in the context of charged black branes. Thus, when $D$ is viewed as a function of $s$, it does not receive a correction of order $k$. However, if $D$ is viewed as a function of the temperature (the energy), then $D$ will change its functional form (at first order in $k$ ) due to the subextensive contribution $E_{c}$ to the total energy. Either way, $\eta=D(\rho+p)$ will receive a correction proportional to $E_{c} \propto k$.

And finally, in the case of charged black holes with scalar fields, we find that $D$, when viewed as a function of $s$, receives a correction of order $k$, since in these cases the term proportional to $k$ in $\eta$ does not equal $E_{c}$, and hence it differs from the contribution $E_{c}$ contained in the total energy. Thus, at first order in $k, \eta=D(s)(\rho+p)$ is not any longer given by (1.2).

In deriving the expressions for $\eta / s$ such as (4.2) we restricted ourselves to corrections of order $k$. Higher corrections in $k$ are in principle also possible. For simplicity, we took the velocity field $u^{\mu}$ of the fluid to be of the specific form (3.7). Our expressions for $\eta / s$ should, however, be independent of this particular choice of the velocity field. We also note that in all the cases considered here, the deviation from $\eta / s=1 /(4 \pi)$ is determined by the coefficient of a $u R u$-term in the associated line element.

In principle, the hydrodynamic energy-momentum tensor may contain additional terms, constructed out of derivatives of the velocity field and/or the curvature tensor on the sphere, that also contribute to $\sigma_{\mu \nu}$ at the same order as the curvature corrections computed in this paper. However, such terms cannot be present for the solutions constructed 
here, neither at the order considered in the paper $\left(\epsilon \delta^{3}\right)$ nor at the next order in derivatives $\left(\epsilon \delta^{4}\right)$. Such terms would have to be constructed from the quantities listed in [33] on page 22 , which contains a comprehensive study of the allowed hydrodynamic quantities classified by their tensorial structure. Since all the quantities appearing in this list either vanish on the solutions considered here or lead to terms that are of higher order in $\epsilon$, it follows that such terms are absent at order $\epsilon$.

\section{Acknowledgments}

We would like to thank J. Erdmenger, M. Haack, R. Loganayagam, S. Minwalla, S. Nampuri, G. Policastro, A. Starinets, E. Witten and A. Yarom for valuable discussions. The work of G. Dall'Agata is partially supported by the Fondazione Cariparo Excellence Grant String-derived supergravities with branes and fluxes and their phenomenological implications and by the ERC Advanced Grant no. 226455 Supersymmetry, Quantum Gravity and Gauge Fields (SUPERFIELDS). The work of G. L. Cardoso is supported in part by the Alexander von Humboldt Foundation, by the Center for Mathematical Analysis, Geometry and Dynamical Systems (CAMGSD) and by Fundação para a Ciência e a Tecnologia (FCT) through the Program Ciência 2008.

\section{A Very special geometry conventions}

The five-dimensional $N=2$ gauged supergravity action is based on a set of real scalar fields $X^{A}$ that satisfy the constraint

$$
\frac{1}{6} C_{A B C} X^{A} X^{B} X^{C}=1
$$

The metric $\mathcal{G}_{A B}$ is given by

$$
\mathcal{G}_{A B}=-\frac{1}{2} C_{A B C} X^{C}+\frac{9}{2} X_{A} X_{B}
$$

where

$$
X_{A}=\frac{1}{6} C_{A B C} X^{B} X^{C} .
$$

Observe that $X^{A} X_{A}=1$ in view of (A.1). In addition,

$$
X_{A} \partial_{i} X^{A}=0
$$

where $X^{A}=X^{A}\left(\varphi^{i}\right)$ and $\partial_{i} X^{A}(\varphi)=\partial X^{A} / \partial \varphi^{i}$. Here the $\varphi^{i}$ denote the physical scalar fields with target-space metric

$$
\mathcal{G}_{i j}=\mathcal{G}_{A B} \partial_{i} X^{A} \partial_{j} X^{B}
$$

A useful relation is

$$
\mathcal{G}_{A B} \partial_{i} X^{B}=-\frac{3}{2} \partial_{i} X_{A}
$$


The potential $V_{\text {pot }}$ is expressed in terms of the superpotential

$$
W=h_{A} X^{A}
$$

and reads

$$
V_{\text {pot }}=\mathfrak{g}^{2}\left(\mathcal{G}^{i j} \partial_{i} W \partial_{j} W-\frac{4}{3} W^{2}\right)=\mathfrak{g}^{2}\left(h_{A} \mathcal{G}^{A B} h_{B}-2 W^{2}\right),
$$

where in the second step we used

$$
\mathcal{G}^{i j} \partial_{i} X^{A} \partial_{j} X^{B}=\mathcal{G}^{A B}-\frac{2}{3} X^{A} X^{B} .
$$

The STU model is based on $X^{1} X^{2} X^{3}=1$, and its metric $\mathcal{G}_{A B}$ is given by

$$
\mathcal{G}_{A B}=\frac{1}{2} \delta_{A B}\left(X^{A}\right)^{-2},
$$

where here there is no summation over $A$.

\section{B Rotating Maxwell black hole in Eddington-Finkelstein type coordi- nates}

The general non-extremal rotating black hole solution in minimal five-dimensional gauged supergravity has been given in [34] in Boyer-Lindquist type coordinates. To linear order in angular velocities $\epsilon \omega_{1}$ and $\epsilon \omega_{2}$ it reads (with $w_{5}=L=k=1$ )

$$
\begin{aligned}
d s^{2}= & \left(-\left(1+a^{2}\right)+\frac{\Sigma}{a^{4}}\right) d t^{2}+\frac{a^{2}}{\Delta_{a}} d a^{2}+a^{2} d \theta^{2}+a^{2}\left(\sin ^{2} \theta d \phi^{2}+\cos ^{2} \theta d \psi^{2}\right) \\
& -\frac{2}{a^{4}}\left(\epsilon \omega_{2} \Sigma+\epsilon \omega_{1} Q a^{2}\right) \cos ^{2} \theta d \psi d t-\frac{2}{a^{4}}\left(\epsilon \omega_{1} \Sigma+\epsilon \omega_{2} Q a^{2}\right) \sin ^{2} \theta d \phi d t, \\
A= & \frac{\sqrt{3} Q}{a^{2}}\left(d t-\epsilon \omega_{1} \sin ^{2} \theta d \phi-\epsilon \omega_{2} \cos ^{2} \theta d \psi\right),
\end{aligned}
$$

where

$$
\Delta_{a}=a^{2}\left(1+a^{2}\right)+\frac{Q^{2}}{a^{2}}-M \quad, \quad \Sigma=M a^{2}-Q^{2} .
$$

The line element in (B.1) can be rewritten in terms of Eddington-Finkelstein type coordinates by applying the following transformations,

$$
d t \rightarrow d t-\frac{a^{2}}{\Delta_{a}} d a \quad, \quad d \phi \rightarrow d \phi-\frac{\epsilon \omega_{1}}{\Delta_{a}}\left(1+a^{2}\right) d a \quad, \quad d \psi \rightarrow d \psi-\frac{\epsilon \omega_{2}}{\Delta_{a}}\left(1+a^{2}\right) d a .
$$

Then, to first order in $\epsilon$, the line element becomes

$$
\begin{aligned}
d s^{2}= & -\frac{\Delta_{a}}{a^{2}} d t^{2}+a^{2} d \Omega_{3}^{2}+2 d t d a \\
& +\frac{2 \epsilon}{a^{4}}\left(\omega_{1} Q^{2}-\omega_{1} M a^{2}-\omega_{2} Q a^{2}\right) \sin ^{2} \theta d t d \phi \\
& +\frac{2 \epsilon}{a^{4}}\left(\omega_{2} Q^{2}-\omega_{2} M a^{2}-\omega_{1} Q a^{2}\right) \cos ^{2} \theta d t d \psi \\
& +2 \epsilon \sin ^{2} \theta\left(\frac{\omega_{2} Q}{\Delta_{a}}-\omega_{1}\right) d a d \phi+2 \epsilon \cos ^{2} \theta\left(\frac{\omega_{1} Q}{\Delta_{a}}-\omega_{2}\right) d a d \psi
\end{aligned}
$$


while the gauge field is still given by (B.1). Rewriting the five-dimensional line element (B.4) in terms of the four-dimensional quantities $u^{\mu}=\left(1,0, \epsilon \omega_{1}, \epsilon \omega_{2}\right), l^{\mu}=$ $\left(0,0,-\epsilon \omega_{2},-\epsilon \omega_{1}\right)$ and $g_{\mu \nu}=\operatorname{diag}\left(-1,1, \sin ^{2} \theta, \cos ^{2} \theta\right)$ yields the line element (3.18) with $\sigma_{\mu \nu}=0$ and $\kappa=-1 /(2 \sqrt{3})$.

\section{A two-charge rotating STU black hole in Eddington-Finkelstein type coordinates}

A rotating version of the static two-charge STU black hole solution (3.32) has been constructed in [36]. To linear order in rotation parameters $\epsilon \omega_{1}$ and $\epsilon \omega_{2}$ it reads

$$
\begin{aligned}
d s^{2}= & H^{-\frac{4}{3}}\left[-\frac{X}{r^{2}} d t^{2}+\frac{2 \epsilon}{r^{2}}\left(X-\frac{f_{3}}{r^{2}}\right)\left(\omega_{1} \sin ^{2} \theta d t d \phi+\omega_{2} \cos ^{2} \theta d t d \psi\right)\right. \\
& \left.+\frac{f_{3}^{2}}{r^{6}}\left(\sin ^{2} \theta d \phi^{2}+\cos ^{2} \theta d \psi^{2}\right)\right]+H^{\frac{2}{3}}\left[\frac{r^{2}}{X} d r^{2}+r^{2} d \theta^{2}\right] \\
H= & 1+\frac{\mu s^{2}}{r^{2}} \\
X= & r^{2}-\mu+\mathfrak{g}^{2}\left(r^{2}+\mu s^{2}\right)^{2} \\
f_{3}= & r^{4}+\mu s^{2} r^{2}
\end{aligned}
$$

where

$$
s=\sinh \delta, \quad c=\cosh \delta .
$$

(Here, $s$ should not be confused with the entropy density in the main text.) The associated gauge potentials are

$$
\begin{aligned}
& A^{1}=A^{2}=\frac{\mu s c}{r^{2} H}\left(d t-\epsilon\left(\omega_{1} \sin ^{2} \theta d \phi+\omega_{2} \cos ^{2} \theta d \psi\right)\right), \\
& A^{3}=\frac{\mu s^{2}}{r^{2}} \epsilon\left(\omega_{2} \sin ^{2} \theta d \phi+\omega_{1} \cos ^{2} \theta d \psi\right) .
\end{aligned}
$$

Setting $\mathfrak{g}^{2}=1, \mu s^{2}=q, \mu s c=Q$ as well as changing the radial coordinate to $a=r H^{\frac{1}{3}}$ and carrying out the transformations

$$
\begin{aligned}
& d t \rightarrow d t-\frac{3 a^{2} H^{\frac{2}{3}}}{X W(a)} d a \\
& d \phi \rightarrow d \phi+\epsilon \omega_{1}\left(d t-\frac{3 H^{-\frac{1}{3}}}{a^{4} f(a) W(a)} d a\right), \quad d \psi \rightarrow d \psi+\epsilon \omega_{2}\left(d t-\frac{3 H^{-\frac{1}{3}}}{a^{4} f(a) W(a)} d a\right)
\end{aligned}
$$

where $f(a)$ is given in (2.10) with $k$ set to $k=1$ and with $\mathrm{e}^{3 U}=H$, yields (C.1) and (C.3) in Eddington-Finkelstein type coordinates to first order in $\epsilon$,

$$
\begin{aligned}
d s^{2}= & -a^{2} f(a) d t^{2}+\frac{6}{W(a)} d a d t-\frac{6}{W(a)} \epsilon\left(\omega_{1} \sin ^{2} \theta d \phi+\omega_{2} \cos ^{2} \theta d \psi\right) d a \\
& +a^{2} d \Omega_{3}^{2}+2 \epsilon\left(a^{2} f(a)+a^{2}-H^{-\frac{1}{3}}\right)\left(\omega_{1} \sin ^{2} \theta d \phi+\omega_{2} \cos ^{2} \theta d \psi\right) d t, \\
A^{1}= & A^{2}=-\frac{Q}{a^{2}} H^{-\frac{1}{3}} u_{\mu} d x^{\mu}, \quad A^{3}=-\frac{q}{a^{2}} H^{\frac{2}{3}} l_{\mu} d x^{\mu},
\end{aligned}
$$


where $W(a)=2 H^{-\frac{1}{3}}+H^{\frac{2}{3}}$ is the superpotential. Then, rewriting the five-dimensional line element (C.5) and gauge potentials (C.3) in terms of the four-dimensional quantities $u^{\mu}=\left(1,0, \epsilon \omega_{1}, \epsilon \omega_{2}\right), l^{\mu}=\left(0,0,-\epsilon \omega_{2},-\epsilon \omega_{1}\right)$ and $g_{\mu \nu}=\operatorname{diag}\left(-1,1, \sin ^{2} \theta, \cos ^{2} \theta\right)$ yields (3.34) with $\sigma_{\mu \nu}=0$.

\section{Three-charge rotating STU black hole with equal rotation parameters in Eddington-Finkelstein coordinates}

A rotating three-charge STU black hole with equal rotation parameters $\omega_{1}=\omega_{2}=\tilde{\omega}$ has been constructed in [37]. To first oder in the rotation parameter $\epsilon \tilde{\omega}$ it reads

$$
\begin{aligned}
d s^{2} & =-\frac{Y}{R^{2}} d t^{2}+\frac{r^{2} R}{Y} d r^{2}+R d \Omega_{3}^{2}-\frac{2 f_{2}}{R^{2}} d t\left(\sin ^{2} \theta d \phi+\cos ^{2} \theta d \psi\right), \\
A^{i} & =\frac{\mu}{r^{2} H_{i}}\left(s_{i} c_{i} d t+\epsilon \tilde{\omega}\left(c_{i} s_{j} s_{k}-s_{i} c_{j} c_{k}\right)\left(\sin ^{2} \theta d \phi+\cos ^{2} \theta d \psi\right)\right), \\
X^{i} & =\frac{R}{r^{2} H_{i}}, \quad i=1,2,3, \quad i \neq j \neq k \neq i,
\end{aligned}
$$

where

$$
\begin{aligned}
& Y=R^{3}+r^{4}-\mu r^{2}, \\
& R=r^{2}\left(\prod_{i=1}^{3} H_{i}\right)^{\frac{1}{3}}, \quad H_{i}=1+\frac{\mu s_{i}^{2}}{r^{2}}, \\
& f_{2}=\epsilon \tilde{\omega}\left(-\gamma R^{3}+\mu\left(\prod_{i} c_{i}-\prod_{i} s_{i}\right) r^{2}+\mu^{2} \prod_{i} s_{i}\right), \\
& s_{i}=\sinh \delta_{i}, \quad c_{i}=\cosh \delta_{i} .
\end{aligned}
$$

Changing the radial coordinate to $a=r \mathrm{e}^{U}=r\left(H_{1} H_{2} H_{3}\right)^{\frac{1}{6}}$ and applying the transformation

$$
d t \rightarrow d t-\frac{3}{W(a) a^{2} f(a)} d a,
$$

yields the line element (D.1) in the form

$$
\begin{aligned}
d s^{2}= & -a^{2} f(a) d t^{2}+\frac{6}{W(a)}\left(d t+\frac{\epsilon \tilde{\omega} h(a)}{a^{2} f}\left(\sin ^{2} \theta d \phi+\cos ^{2} \theta d \psi\right)\right) d a \\
& +a^{2} d \Omega_{3}^{2}-2 \epsilon \tilde{\omega} h(a)\left(\sin ^{2} \theta d \phi+\cos ^{2} \theta d \psi\right) d t,
\end{aligned}
$$

where

$$
h(a)=-\gamma a^{2}+\frac{\mu}{a^{2}} \mathrm{e}^{-2 U}\left(\prod_{i} c_{i}-\prod_{i} s_{i}\right)+\frac{\mu^{2}}{a^{4}} \prod_{i} s_{i} .
$$

For later convenience we define $\omega=\gamma \tilde{\omega}$ and $\tilde{h}=\gamma^{-1} h$ such that $\omega \tilde{h}=\tilde{\omega} h$. Then carrying out the transformations

$$
d \phi \rightarrow d \phi+\epsilon \omega\left(d t-\frac{3\left(a^{2} f(a)+\tilde{h}(a)\right)}{a^{4} f(a) W(a)} d a\right), d \psi \rightarrow d \psi+\epsilon \omega\left(d t-\frac{3\left(a^{2} f(a)+\tilde{h}(a)\right)}{a^{4} f(a) W(a)} d a\right)
$$


yields (D.1) in Eddington-Finkelstein type coordinates to first order in $\epsilon$,

$$
\begin{aligned}
d s^{2}= & -a^{2} f(a) d t^{2}+\frac{6}{W(a)}\left(d t-\epsilon \omega\left(\sin ^{2} \theta d \phi+\cos ^{2} \theta d \psi\right)\right) d a \\
& +a^{2} d \Omega_{3}^{2}+2 \epsilon \omega\left(a^{2} f(a)+a^{2}-\left(a^{2} f(a)+\tilde{h}(a)\right)\right)\left(\sin ^{2} \theta d \phi+\cos ^{2} \theta d \psi\right) d t, \\
A^{i}= & \frac{\mu}{a^{2} H_{i}} e^{2 U}\left(s_{i} c_{i} d t+\epsilon \frac{\omega}{\gamma}\left(c_{i} s_{j} s_{k}-s_{i} c_{j} c_{k}\right)\left(\sin ^{2} \theta d \phi+\cos ^{2} \theta d \psi\right)\right), \\
X^{i}= & \frac{1}{H_{i}}\left(\prod_{i=1}^{3} H_{i}\right)^{\frac{1}{3}}, \quad i=1,2,3, \quad i \neq j \neq k \neq i .
\end{aligned}
$$

The line element in (D.7) is related to the various line elements used in the main text, as follows. Let us first consider the stationary limit of the Maxwell solution (3.18) with $\omega_{1}=\omega_{2}=\omega$. It is obtained from (D.7) by setting $\delta_{1}=\delta_{2}=\delta_{3}=\delta, W(a)=3$ and $\Delta_{a}=f a^{4}$ with $f$ given by $f(a)=1+k / a^{2}-M / a^{4}+Q^{2} / a^{6}$. Then the function $h$ becomes (with $s_{i}=s, c_{i}=c$ )

$$
h(a)=-\gamma a^{2}+\frac{\mu}{a^{2}} \mathrm{e}^{-2 U}\left(c^{3}-s^{3}\right)+\frac{\mu^{2}}{a^{4}} s^{3},
$$

which can also be written as

$$
h(a)=\gamma \tilde{h}(a)=(c-s)\left(-a^{2}+\frac{\mu}{a^{2}} \mathrm{e}^{-2 U}\left(c^{2}+s^{2}+c s\right)+\frac{\mu^{2}}{a^{4}} s^{3}(c+s)\right) .
$$

Setting $M=\mu+2 \mu s^{2}, Q=\mu s c$ and $\mathrm{e}^{-2 U}=\left(a^{2}-\mu s^{2}\right) / a^{2}$ gives

$$
\tilde{h}(a)=-a^{2} f(a)+1+\frac{Q}{a^{2}} .
$$

The terms in this expression are related as follows to the ones in (3.18): the second term is the coefficient of the $u R u$-term, while the third term is the coefficient of the $u l$-term.

Next, let us consider the stationary limit of the two-charge solution (3.34). It is obtained from (D.7) by setting $\delta_{1}=\delta_{2}=\delta, \delta_{3}=0, \gamma=1$ and $H=\mathrm{e}^{3 U}$. Then the function $h$ becomes

$$
h(a)=\tilde{h}(a)=-a^{2} f(a)+\mathrm{e}^{-U},
$$

with $f$ given by (2.10). In this expression, the second term is the coefficient of the $u R u$ term in (3.34).

And finally, the stationary limit of the one-charge solution (3.42) is obtained from (D.7) by setting $\delta_{1}=\delta$ and $\delta_{2}=\delta_{3}=0$. Now the function $h$ reads (with $c_{1}=c$ )

$$
h(a)=-\gamma a^{2}+\frac{\mu}{a^{2}} \mathrm{e}^{-2 U} c .
$$

This can be written as

$$
h(a)=\gamma \tilde{h}(a)=\frac{1}{c}\left(-a^{2}+\frac{\mu}{a^{2}} \mathrm{e}^{-2 U} c^{2}\right) .
$$

Setting $\gamma=c^{-1}$, and with $H=\mathrm{e}^{6 U}$, we obtain

$$
\tilde{h}(a)=-a^{2} f(a)+\mathrm{e}^{2 U},
$$

with $f$ given by (2.10). In this expression, the second term is the coefficient of the $u R u$ term in (3.42). 


\section{E Boundary energy-momentum tensor for the STU black hole solution (3.34)}

Here we compute the boundary energy-momentum for the STU black hole carrying two equal charges. A similar calculation applies to the other cases discussed in the main text, namely no charge (the Schwarzschild case), one non-vanishing charge and three equal charges (the Maxwell case).

The boundary energy-momentum tensor is given by [30-32]

$$
8 \pi G_{5}\left\langle T_{\mu \nu}\right\rangle=\lim _{a \rightarrow \infty}\left[a^{2}\left(K_{\mu \nu}-K \gamma_{\mu \nu}-\frac{W(a)}{L} \gamma_{\mu \nu}+\frac{L}{2} G_{\mu \nu}\right)\right],
$$

where the boundary metric $\gamma_{\mu \nu}$ is read off from the bulk metric written in the form

$$
d s^{2}=N^{2} d a^{2}+\gamma_{\mu \nu}\left(d x^{\mu}+n^{\mu} d a\right)\left(d x^{\nu}+n^{\nu} d a\right),
$$

$G_{\mu \nu}=R_{\mu \nu}[\gamma]-\frac{1}{2} \gamma_{\mu \nu} R[\gamma]$ is the four-dimensional Einstein tensor of $\gamma_{\mu \nu}$, and the extrinsic curvature tensor is given by [29]

$$
K_{\mu \nu}=-\frac{1}{2 N}\left(\partial_{a} \gamma_{\mu \nu}-\nabla_{\mu}[\gamma] n_{\nu}-\nabla_{\nu}[\gamma] n_{\mu}\right)
$$

with $K=\gamma^{\mu \nu} K_{\mu \nu}$. Here $n_{\mu}=\gamma_{\mu \nu} n^{\nu}$, and $W(a)$ is the superpotential.

Imposing the tracelessness of $T_{\mu \nu}$ results in $K=-4 W(a) /(3 L)-L R[\gamma] / 6$, and reinserting this into (E.1) yields

$$
8 \pi G_{5}\left\langle T_{\mu \nu}\right\rangle=\lim _{a \rightarrow \infty}\left[a^{2}\left(K_{\mu \nu}+\frac{W(a)}{3 L} \gamma_{\mu \nu}+\frac{L}{2}\left(R_{\mu \nu}[\gamma]-\frac{1}{6} \gamma_{\mu \nu} R[\gamma]\right)\right)\right] .
$$

In the following we set $L=1$.

Comparing (E.2) with the line element (3.34) for the deformed STU black hole, and using (3.39), we infer that for large $a$,

$$
\begin{aligned}
n_{\mu}= & -\frac{3}{W(a)} u_{\mu} \quad, \quad N^{2}=-\frac{9}{W(a)^{2}} \gamma^{\mu \nu} u_{\mu} u_{\nu}, \\
\gamma_{\mu \nu}= & a^{2} g_{\mu \nu}-\left(\mathrm{e}^{-4 U} k-\mathrm{e}^{-2 U} \frac{\mu}{a^{2}}\right) u_{\mu} u_{\nu}+\frac{1}{2} \mathrm{e}^{-U}\left(u_{\mu} R_{\nu \lambda} u^{\lambda}+u_{\nu} R_{\mu \lambda} u^{\lambda}\right) \\
& +\left(2 a-\frac{\eta}{2 a^{2}}\right) \sigma_{\mu \nu} .
\end{aligned}
$$

Here $W(a) \approx 3+q^{2} /\left(3 a^{4}\right)$ and the exponential functions $\mathrm{e}^{-\chi U}$ in $\gamma_{\mu \nu}$ behave as $\mathrm{e}^{-\chi U} \approx$ $1-\chi q /\left(3 a^{2}\right)$ so that

$$
\begin{aligned}
\gamma_{\mu \nu}= & a^{2} g_{\mu \nu}-\left(k-\frac{w_{5} M}{a^{2}}\right) u_{\mu} u_{\nu}+\frac{1}{2}\left(1-\frac{q}{3 a^{2}}\right)\left(u_{\mu} R_{\nu \lambda} u^{\lambda}+u_{\nu} R_{\mu \lambda} u^{\lambda}\right) \\
& +\left(2 a-\frac{\eta}{2 a^{2}}\right) \sigma_{\mu \nu}
\end{aligned}
$$

where $w_{5} M=\mu+\frac{4}{3} k q$ is the physical mass. At first order in $\epsilon$ and at large $a$, the inverse metric $\gamma^{\mu \nu}$ is then given by

$$
\gamma^{\mu \nu}=\frac{1}{a^{2}} g^{\mu \nu}+\frac{k}{a^{4}} u^{\mu} u^{\nu}-\frac{1}{2 a^{4}}\left(u^{\mu} R_{\lambda}^{\nu} u^{\lambda}+u^{\nu} R_{\lambda}^{\mu} u^{\lambda}\right)-\frac{2}{a^{3}} \sigma^{\mu \nu},
$$


where the indices on the right hand side are raised with the metric $g^{\mu \nu}$.

Computing the terms in (E.4) for large $a$ and to first order in $\epsilon$, we obtain

$$
\begin{aligned}
W(a)= & 3+\frac{q^{2}}{3 a^{4}}, \\
N^{-1}= & a+\frac{k}{2 a}-\frac{k^{2}}{8 a^{3}}-\frac{w_{5} M}{2 a^{3}}+\frac{q^{2}}{9 a^{3}}, \\
-\frac{1}{2 N} \partial_{a} \gamma_{\mu \nu}+\frac{W(a)}{3} \gamma_{\mu \nu}= & \frac{1}{2 a^{2}}\left(\frac{k^{2}}{4} g_{\mu \nu}+w_{5} M\left(g_{\mu \nu}+4 u_{\mu} u_{\nu}\right)-2 \eta \sigma_{\mu \nu}\right) \\
& -\frac{k}{2}\left(g_{\mu \nu}+2 u_{\mu} u_{\nu}\right)+\left(a-\frac{k}{2 a}\right) \sigma_{\mu \nu} \\
& +\left(\frac{1}{2}-\frac{q}{3 a^{2}}\right)\left(u_{\mu} R_{\nu \lambda} u^{\lambda}+u_{\nu} R_{\mu \lambda} u^{\lambda}\right), \\
\Gamma_{\mu \beta}[\gamma] n_{\nu}= & -\nabla_{\mu}^{\gamma} u_{\nu}, \frac{1}{a} g^{\gamma \lambda}\left(\nabla_{\alpha} \sigma_{\lambda \beta}+\nabla_{\beta} \sigma_{\alpha \lambda}-\nabla_{\lambda} \sigma_{\alpha \beta}\right), \\
R_{\mu \nu}[\gamma]= & R_{\mu \nu}+\frac{4 k}{a} \sigma_{\mu \nu}, \\
R[\gamma]= & \frac{R}{a^{2}}, \\
R_{\mu \nu}[\gamma]-\frac{1}{6} \gamma_{\mu \nu} R[\gamma]= & R_{\mu \nu}-k g_{\mu \nu}+\frac{k^{2}}{a^{2}} u_{\mu} u_{\nu} \\
& -\frac{k}{2 a^{2}}\left(u_{\mu} R_{\nu \lambda} u^{\lambda}+u_{\nu} R_{\mu \lambda} u^{\lambda}\right)+\frac{2 k}{a} \sigma_{\mu \nu} .
\end{aligned}
$$

Inserting these expressions into (E.4) yields the energy-momentum tensor (3.40).

Open Access. This article is distributed under the terms of the Creative Commons Attribution Noncommercial License which permits any noncommercial use, distribution, and reproduction in any medium, provided the original author(s) and source are credited.

\section{References}

[1] G. Policastro, D.T. Son and A.O. Starinets, The shear viscosity of strongly coupled $N=4$ supersymmetric Yang-Mills plasma, Phys. Rev. Lett. 87 (2001) 081601 [hep-th/0104066] [SPIRES].

[2] G. Policastro, D.T. Son and A.O. Starinets, From AdS/CFT correspondence to hydrodynamics, JHEP 09 (2002) 043 [hep-th/0205052] [SPIRES].

[3] S. Bhattacharyya, V.E. Hubeny, S. Minwalla and M. Rangamani, Nonlinear fluid dynamics from gravity, JHEP 02 (2008) 045 [arXiv:0712.2456] [SPIRES].

[4] A. Buchel and J.T. Liu, Universality of the shear viscosity in supergravity, Phys. Rev. Lett. 93 (2004) 090602 [hep-th/0311175] [SPIRES].

[5] A. Buchel, On universality of stress-energy tensor correlation functions in supergravity, Phys. Lett. B 609 (2005) 392 [hep-th/0408095] [SPIRES].

[6] J. Mas, Shear viscosity from R-charged AdS black holes, JHEP 03 (2006) 016 [hep-th/0601144] [SPIRES]. 
[7] D.T. Son and A.O. Starinets, Hydrodynamics of R-charged black holes, JHEP 03 (2006) 052 [hep-th/0601157] [SPIRES].

[8] K. Maeda, M. Natsuume and T. Okamura, Viscosity of gauge theory plasma with a chemical potential from AdS/CFT, Phys. Rev. D 73 (2006) 066013 [hep-th/0602010] [SPIRES].

[9] P. Benincasa, A. Buchel and R. Naryshkin, The shear viscosity of gauge theory plasma with chemical potentials, Phys. Lett. B 645 (2007) 309 [hep-th/0610145] [SPIRES].

[10] C. Eling, Hydrodynamics of spacetime and vacuum viscosity, JHEP 11 (2008) 048 [arXiv: 0806.3165] [SPIRES].

[11] J. Erdmenger, M. Haack, M. Kaminski and A. Yarom, Fluid dynamics of R-charged black holes, JHEP 01 (2009) 055 [arXiv: 0809.2488] [SPIRES].

[12] N. Banerjee et al., Hydrodynamics from charged black branes, arXiv:0809.2596 [SPIRES].

[13] N. Iqbal and H. Liu, Universality of the hydrodynamic limit in AdS/CFT and the membrane paradigm, Phys. Rev. D 79 (2009) 025023 [arXiv:0809.3808] [SPIRES].

[14] M. Torabian and H.-U. Yee, Holographic nonlinear hydrodynamics from AdS/CFT with multiple/non-Abelian symmetries, JHEP 08 (2009) 020 [arXiv: 0903.4894] [SPIRES].

[15] S. Bhattacharyya, S. Lahiri, R. Loganayagam and S. Minwalla, Large rotating AdS black holes from fluid mechanics, JHEP 09 (2008) 054 [arXiv:0708.1770] [SPIRES].

[16] M.M. Caldarelli, O.J.C. Dias, R. Emparan and D. Klemm, Black holes as lumps of fluid, JHEP 04 (2009) 024 [arXiv:0811.2381] [SPIRES].

[17] M.M. Caldarelli, O.J.C. Dias and D. Klemm, Dyonic AdS black holes from magnetohydrodynamics, JHEP 03 (2009) 025 [arXiv:0812.0801] [SPIRES].

[18] V. Cardoso and O.J.C. Dias, Bifurcation of plasma balls and black holes to Lobed configurations, JHEP 04 (2009) 125 [arXiv:0902.3560] [SPIRES].

[19] E.P. Verlinde, On the holographic principle in a radiation dominated universe, hep-th/0008140 [SPIRES].

[20] I. Savonije and E.P. Verlinde, CFT and entropy on the brane, Phys. Lett. B 507 (2001) 305 [hep-th/0102042] [SPIRES].

[21] G. Koutsoumbas, E. Papantonopoulos and G. Siopsis, Shear viscosity and Chern-Simons diffusion rate from hyperbolic horizons, Phys. Lett. B 677 (2009) 74 [arXiv:0809.3388] [SPIRES].

[22] R. Loganayagam, Entropy current in conformal hydrodynamics, JHEP 05 (2008) 087 [arXiv: 0801.3701] [SPIRES].

[23] S. Bhattacharyya, R. Loganayagam, I. Mandal, S. Minwalla and A. Sharma, Conformal nonlinear fluid dynamics from gravity in arbitrary dimensions, JHEP 12 (2008) 116 [arXiv:0809.4272] [SPIRES].

[24] K. Behrndt, M. Cvetič and W.A. Sabra, Non-extreme black holes of five dimensional $N=2$ AdS supergravity, Nucl. Phys. B 553 (1999) 317 [hep-th/9810227] [SPIRES].

[25] M. Günaydin, G. Sierra and P.K. Townsend, Gauging the D $=5$ Maxwell-Einstein supergravity theories: more on Jordan algebras, Nucl. Phys. B 253 (1985) 573 [SPIRES].

[26] G.L. Cardoso and V. Grass, On five-dimensional non-extremal charged black holes and FRW cosmology, Nucl. Phys. B 803 (2008) 209 [arXiv:0803.2819] [SPIRES]. 
[27] W.A. Sabra, General BPS black holes in five dimensions, Mod. Phys. Lett. A 13 (1998) 239 [hep-th/9708103] [SPIRES].

[28] H. Elvang, D.Z. Freedman and H. Liu, From fake supergravity to superstars, JHEP 12 (2007) 023 [hep-th/0703201] [SPIRES].

[29] M. Haack and A. Yarom, Nonlinear viscous hydrodynamics in various dimensions using AdS/CFT, JHEP 10 (2008) 063 [arXiv:0806.4602] [SPIRES].

[30] V. Balasubramanian and P. Kraus, A stress tensor for anti-de Sitter gravity, Commun. Math. Phys. 208 (1999) 413 [hep-th/9902121] [SPIRES].

[31] R. Emparan, C.V. Johnson and R.C. Myers, Surface terms as counterterms in the AdS/CFT correspondence, Phys. Rev. D 60 (1999) 104001 [hep-th/9903238] [SPIRES].

[32] A. Batrachenko, J.T. Liu, R. McNees, W.A. Sabra and W.Y. Wen, Black hole mass and Hamilton-Jacobi counterterms, JHEP 05 (2005) 034 [hep-th/0408205] [SPIRES].

[33] S. Bhattacharyya et al., Forced fluid dynamics from gravity, JHEP 02 (2009) 018 [arXiv:0806.0006] [SPIRES].

[34] Z.W. Chong, M. Cvetič, H. Lü and C.N. Pope, General non-extremal rotating black holes in minimal five- dimensional gauged supergravity, Phys. Rev. Lett. 95 (2005) 161301 [hep-th/0506029] [SPIRES].

[35] S. Minwalla, Nonlinear fluid dynamics from gravity, http://ph-dep-th.web.cern.ch/ph-dep-th/content2/workshops/strings2008/ (2008).

[36] Z.W. Chong, M. Cvetič, H. Lü and C.N. Pope, Five-dimensional gauged supergravity black holes with independent rotation parameters, Phys. Rev. D 72 (2005) 041901 [hep-th/0505112] [SPIRES].

[37] M. Cvetič, H. Lü and C.N. Pope, Charged rotating black holes in five dimensional $U_{1}^{3}$ gauged $N=2$ supergravity, Phys. Rev. D 70 (2004) 081502 [hep-th/0407058] [SPIRES].

[38] Z.W. Chong, M. Cvetič, H. Lü and C.N. Pope, Non-extremal rotating black holes in five-dimensional gauged supergravity, Phys. Lett. B 644 (2007) 192 [hep-th/0606213] [SPIRES]. 